\title{
Global Chassis Control Based on Inverse Vehicle Dynamics Models
}

\author{
Johan Andreasson and Tilman Bünte \\ (Received 00 Month 200x; In final form 00 Month 200x)
}

\begin{abstract}
This work proposes to approach Global Chassis Control (GCC) by means of model inversion based feedforward with allocation directly on the actuator commands. The available degrees of freedom are used to execute the desired vehicle motion while minimizing the utilization of the tyre's grip potential. This is done by sampled constrained least-squares optimization of the linearized problem. For compensation of model errors and external disturbances, high gain feedback is applied by means of an Inverse Disturbance Observer. The presented method is applied on a comparison of eight vehicles with different configuration of actuators for steer, drive, brake and load distribution. The approach shows a transparent and effective way to deal with the complex issue of GCC in a unitized way. It gives both a base for controller design and a structured way to compare different configurations. In practice, the transparency supports automatic on-board reconfiguration in case of actuator hardware failure.
\end{abstract}

\section{Introduction}

As the number of vehicle dynamics control (VDC) subsystems grows, integration of these gets more and more important. For the coordination of the involved actuators, there is a variety of names and here it is referred to as Global Chassis Control (GCC).

Various approaches for GCC have already been developed and it is seen that a main issue is the huge amount of combinations of possibilities to affect the vehicle's motion, depending on the vehicle's actuator configuration. Some work is based on various approaches considering properties of a given combination of actuators [1-3].For these approaches, controllers have to be designed and calibrated for each single configuration evaluated. As a result, the assessment is limited to a few combinations, often as an extension to existing VDC systems like ESP, with a limited number of additional actuators.

Other GCC concepts start from scratch while exploiting the full synergetic potential of the actuators being available. One example in [4] where each

KTH Vehicle Dynamics, SE-100 44 Stockholm, Sweden, e-mail: nahoj@kth.se, phone: +46 879077 14, fax: +4687909304

Institute of Robotics and Mechatronics, DLR, D-82230, Wessling, Germany, e-mail: Tilman.Buente@dlr.de, phone: +49 8153 28-1627 fax: +49 8153 28-4-1627 


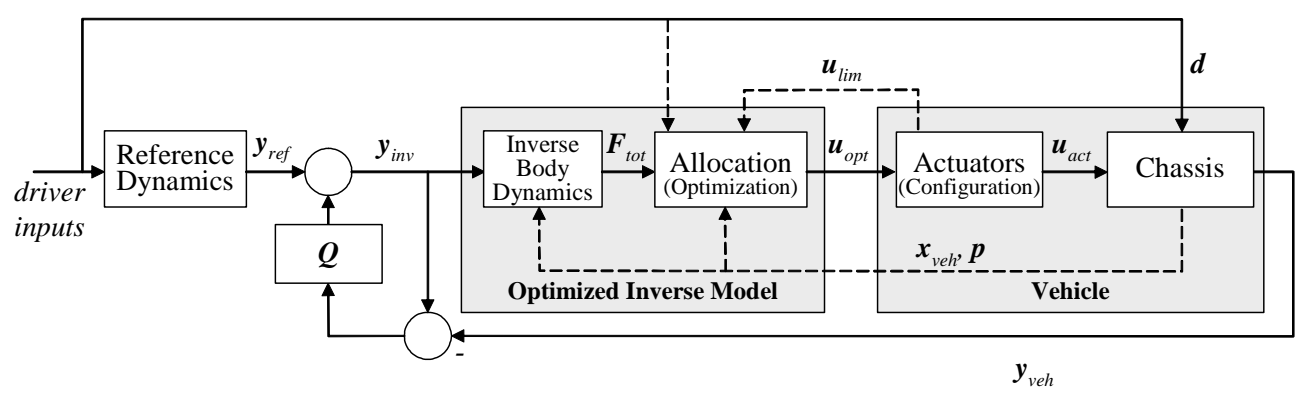

Figure 1. Global Chassis Control (GCC) based on Inverse Disturbance Observer (IDOB) for feedback and model inversion feedforward.

wheel is assumed to be individually steered and driven. This requires allocation of tyre forces within their friction limits and optimization is used for this. A generic force allocation method is presented in $[5,6]$ where actuators are abstracted to their effect on the tyres' potential to generate force, giving an optimization problem with nonlinear constraints that adapts to the current vehicle configuration.

The approach introduced here is based on model inversion based feedforward control with allocation directly on the actuator commands by optimization, combined with high gain feedback.

\section{Global Chassis Control Structure}

Within this work, the aim is to provide both a qualified GCC structure and an effective design methodology that are generic in the sense that they are invariant for different actuator configurations and even for diverse types of vehicles (e.g. with different number of wheels $n$ ). Figure 1 shows the suggested GCC structure based on Inverse Disturbance Observer (IDOB) design [7, 8]. It consists of a feedforward part, which is an approximate inverse of the vehicle, and a so-called $Q$-loop that provides high gain feedback. This control structure has been shown to be efficient for generic nonlinear MIMO tracking tasks [8].

In the feedforward part, the driver input is used by Reference Dynamics to calculate a reference motion, $\boldsymbol{y}_{\text {ref }}$, that together with the feedback determines the motion for the Optimized Inverse Model. As indicated in Figure 1, the inverse model consists of two steps; Inverse Body Dynamics and Allocation. For given vehicle mass and inertia, the vector $\boldsymbol{F}_{\text {tot }}$ of total torques and forces to be generated by the chassis depends on $\boldsymbol{y}_{\text {inv }}$ in a unique way and is computed by an inverted lean model. Measured or observed vehicle states, $\boldsymbol{x}_{v e h}$, can be used to directly set states of this inverse model instead of internal integration. This corresponds to the technique of dynamic inversion which is established 
e.g. in nonlinear control of aircraft [9].

However, the task of realizing $\boldsymbol{F}_{\text {tot }}$ by appropriate actuator commands may have manifold solutions [4]. The choice of a specific solution is here referred to as control allocation where the number of degrees of freedom depends on the effective vehicle actuation configuration. Furthermore, the number is potentially decreased in the situation where tyres and/or actuators are at their limit. Optimization is used to exploit the present degrees of freedom which are represented by available actuator commands and is described in more detail in Section 3.

In general, GCC may cover intentional effects on roll, pitch and bounce dynamics and it is also possible to add actuators for e.g. improved down force (by means of aerodynamic control surfaces) and camber control. For the sake of clarity, the presentation is here limited to cover more common actuators and their effects on the horizontal motion. For a vehicle with $n$ wheels, $\boldsymbol{u}$ is defined as

$$
\boldsymbol{u}=\left[\tau_{1}, \delta_{1}, f_{z 1}, \tau_{2}, \delta_{2}, f_{z 2}, \ldots, \tau_{n}, \delta_{n}, f_{z n}\right]^{T}
$$

corresponding to Table 1 , where $\delta_{i}$ denotes steering angles, $\tau_{i}$ drive/brake torques and $f_{z i}$ tyre loads. For certain configurations (e.g. conventional front steering), some driver inputs $\boldsymbol{d}$ may directly act on the vehicle.

Table 1. A subset of conceivable actuator configurations for a four wheeled car. Note that for a general case, there is neither need to limit the setup for four-wheeled vehicles nor for these actuators. Indices refer to wheels (1: front left, 2: front right, 3: rear left, 4: rear right).

\begin{tabular}{rll}
\hline configuration & actuators & description \\
\hline $\mathrm{A}:$ & $\delta_{1}=\delta_{2}$ & Active front axle steering \\
$\mathrm{B}:$ & $\delta_{1}=\delta_{2}, \delta_{3}=\delta_{4}$ & Active front and rear axle steering \\
$\mathrm{C}:$ & $\delta_{1}, \delta_{2}, \delta_{3}, \delta_{4}$ & Individual wheel steering \\
\hline $1:$ & $\sum \tau_{i}$ & Vehicle speed \\
$2 \mathrm{a}:$ & $\tau_{1}<0, \tau_{2}<0, \tau_{3}, \tau_{4}$ & Individual brake and rear wheel drive \\
$2 \mathrm{~b}:$ & $\tau_{1}, \tau_{2}, \tau_{3}<0, \tau_{4}<0$ & Individual brake and front wheel drive \\
$3:$ & $\tau_{1}, \tau_{2}, \tau_{3}, \tau_{4}$ & Individual drive and brake \\
\hline $\mathrm{I}:$ & $f\left(f_{z 1}, f_{z 2}, f_{z 3}, f_{z 4}\right)=0$ & Variable tyre load distribution \\
\hline $0:$ & - & No actuator/control
\end{tabular}

In this work, instead of the more commonly used motion coordinates resolved in the vehicle frame, the desired motion is given by a trajectory referring to the vehicle's center of gravity together with a given speed profile [5]. This yields a reference acceleration in the direction of travel $a_{\text {long,ref }}$ and a reference lateral acceleration $a_{\text {lat,ref }}=\rho v^{2}$ where $\rho$ is the actual trajectory curvature and $v$ is 
the actual speed. The horizontal motion becomes unique when also the side slip angle is given and the vector $\boldsymbol{y}_{r e f}$ is thus completed by the side slip angle, giving $\boldsymbol{y}_{\text {ref }}=\left[a_{\text {long }}, a_{\text {lat }}, \beta\right]_{\text {ref }}^{\mathrm{T}}$.

A transfer function matrix $\mathbf{Q}=\operatorname{diag}\left(Q_{a_{\text {long }}}, Q_{a_{\text {lat }}}, Q_{\beta}\right)$ is used in a subordinate loop yielding high gain for the feedback control, where $Q_{j}$ are unity gain low pass filters. This results in $\boldsymbol{y}_{v e h}$ tracking $\boldsymbol{y}_{r e f}$. The bandwidth of the $Q_{j}$-filters are the main tuning parameters for performance and stability of the feedback control [8].

\section{Allocation}

Depending on configuration, the desired motion can be fulfilled to a different extent. Further criteria for allocation of horizontal tyre forces via actuator commands may be incorporated, resulting in an optimization problem of multiple criteria. In the sequel, the optimization algorithm is assumed to be executed at a fixed sample interval $\Delta t$.

The main task is to achieve the desired total torques and forces acting on the chassis, yielding a linear problem

$$
\mathbf{B} \boldsymbol{f}(\boldsymbol{u}, \boldsymbol{p})=\boldsymbol{F}_{t o t}=\left[\begin{array}{c}
M_{z} \\
F_{x} \\
F_{y}
\end{array}\right]
$$

where the matrix $\mathbf{B}$ depends on the wheel locations, $\boldsymbol{f}(\boldsymbol{u}, \boldsymbol{p})$ is the vector of horizontal forces at each wheel, depending on the actuator commands $\boldsymbol{u}$ and the parameter vector $\boldsymbol{p}$. For simplicity, $\boldsymbol{p}$ is omitted in the sequel notation.

Equation 2 only requires the motion to be fulfilled, which for many configurations yields an under-determined problem that therefore has manifold solutions as mentioned earlier. Additional criteria are added to benefit from these degrees of freedom as explained below, giving in most cases an overdetermined problem is yielded which can be solved for $\boldsymbol{u}$ in the least-squares sense.

To avoid manifold solutions, an additional vector valued criterion is employed to keep the utilization of the tyre grip potential at every wheel low (similar to [4]) and preferably equal, giving

$$
\min _{\boldsymbol{u}}\left|\boldsymbol{\eta}^{\prime}\right|_{2} \text { with } \boldsymbol{\eta}^{\prime}(\boldsymbol{u})=\boldsymbol{\eta}(\boldsymbol{u})-\zeta \eta_{\min } \cdot \mathbf{1}
$$

At the wheel $i$, the degree of utilization of the tyre grip potential is denoted $\eta_{i}$ with $0 \leq \eta_{i} \leq 1, \boldsymbol{\eta}=\left[\eta_{1}, \eta_{2}, \ldots, \eta_{n}\right]^{\mathrm{T}}$ and $\mathbf{1}=[1,1, \ldots, 1]^{\mathrm{T}}$. The lowest $\eta_{i}$ preceding the actual optimization sample is denoted $\eta_{\min }$. It is used as a 
reference value for the actual optimization sample together with a heuristically adjustable decay parameter $\zeta$ with $0 \leq \zeta<1$. The latter directs the optimization towards lower $\eta$-values.

The main motivation for the auxiliary criterion is primarily to keep the safety margin high. Additional benefits are lower fuel consumption and lower tyre wear.

Equations $(2,3)$ are similar to control allocation problems for flight control, but while aircrafts normally have to deal with componentwise rudder deflections, vehicles equipped with tyres instead have nonlinear, coupled forces. In [5] this is dealt with by assigning nonlinear constraints on $\boldsymbol{f}(\boldsymbol{u})$ and then applying inverse tyre models to find $\boldsymbol{u}$. Other optimization-based approaches try to solve the nonlinear optimization problem for simple cases where it is shown to be convex [4]. Here, it is assumed that abrupt changes of actuator positions are unwanted. Thus, the optimization problem can be linearized while putting adequate constraints on actuator rates, yielding smooth actuator commands which evolve along an (at least locally) optimal solution. This approach has already been successfully applied to real time control of industrial robots [10].

Thus, $\boldsymbol{u}$ is directly allocated by a linearization of equations $(2,3)$ about the current operating point $\boldsymbol{u}^{*}$, giving

$$
\left[\begin{array}{c}
\boldsymbol{f}(\boldsymbol{u}) \\
\boldsymbol{\eta}^{\prime}(\boldsymbol{u})
\end{array}\right] \approx\left[\begin{array}{l}
\boldsymbol{f}\left(\boldsymbol{u}^{*}\right) \\
\boldsymbol{\eta}\left(\boldsymbol{u}^{*}\right)
\end{array}\right]+\frac{\partial}{\partial \boldsymbol{u}}\left[\begin{array}{l}
\boldsymbol{f}(\boldsymbol{u}) \\
\boldsymbol{\eta}(\boldsymbol{u})
\end{array}\right]_{\boldsymbol{u}=\boldsymbol{u}^{*}} \tilde{\boldsymbol{u}}
$$

using $\tilde{\boldsymbol{u}}=\boldsymbol{u}-\boldsymbol{u}^{*}$. The complete allocation problem covering all criteria and constraints is to find $\tilde{\boldsymbol{u}}=\tilde{\boldsymbol{u}}_{\text {opt }}$ such that

$$
\min _{\tilde{\boldsymbol{u}}}(\mathbf{A} \tilde{\boldsymbol{u}}-\boldsymbol{b})^{\mathrm{T}} \mathbf{W}^{\mathrm{T}} \mathbf{W}(\mathbf{A} \tilde{\boldsymbol{u}}-\boldsymbol{b})
$$

with a suitable diagonal weighting matrix $\mathbf{W}$ and subject to the constraints

$$
\begin{aligned}
\mathrm{C} \tilde{\boldsymbol{u}}-\boldsymbol{d} & =\mathbf{0} \\
\mathrm{G} \tilde{\boldsymbol{u}}-\boldsymbol{h} & \geq \mathbf{0} .
\end{aligned}
$$

Here,

$$
\mathbf{A}=\left[\begin{array}{ll}
\mathbf{B} & \mathbf{0} \\
\mathbf{0} & \mathbf{I}
\end{array}\right] \frac{\partial}{\partial \boldsymbol{u}}\left[\begin{array}{l}
\boldsymbol{f}(\boldsymbol{u}) \\
\boldsymbol{\eta}(\boldsymbol{u})
\end{array}\right]_{\boldsymbol{u}=\boldsymbol{u}^{*}}, \text { and } \boldsymbol{b}=\left[\begin{array}{c}
\boldsymbol{F}_{t o t} \\
\zeta \eta_{\min } \cdot \mathbf{1}_{n \times 1}
\end{array}\right]-\left[\begin{array}{cc}
\mathbf{B} & \mathbf{0} \\
\mathbf{0} & \mathbf{I}
\end{array}\right]\left[\begin{array}{l}
\boldsymbol{f}\left(\boldsymbol{u}^{*}\right) \\
\boldsymbol{\eta}\left(\boldsymbol{u}^{*}\right)
\end{array}\right] .
$$

Additional criteria like e.g. comfort relevant issues could be included in the same manner by adding rows in $\mathbf{A}, \boldsymbol{b}$, and $\mathbf{W}$. 
Equation 5 with $\boldsymbol{d}=\mathbf{0}$ opens up for convenient adaptation to the actual vehicle configuration, exemplified here by a front rack steering mechanism used in configurations A and B in Table 1. The representing constraint couples the steering angles of the front right and rear wheel, described as $f_{\text {rack }}\left(\delta_{1}, \delta_{2}\right)=0$. This equation is linearized about the current operating point, contributing with a row $\left[0, \partial f_{\text {rack }} / \partial \delta_{1}, 0,0, \partial f_{\text {rack }} / \partial \delta_{2}, 0,0,0,0, \ldots\right]$ in $\mathbf{C}$. Other constraints are added correspondingly by supplying their relevant partial derivatives, giving a new row for each constraint. Additionally, for all configurations in the planar case studied here, three constraints for tyre loads are present representing the force and torque balances for roll, pitch and bounce.

By means of equation 6 with $\mathbf{G}=[-\mathbf{I}, \mathbf{I}]^{\mathrm{T}}$ both actuator position and rate limitations can be considered with

$$
\boldsymbol{h}=\left[-\min \left(\boldsymbol{u}_{\max }-\boldsymbol{u}^{*}, \Delta t \cdot \dot{\boldsymbol{u}}_{\max }\right), \max \left(\boldsymbol{u}_{\min }-\boldsymbol{u}^{*}, \Delta t \cdot \dot{\boldsymbol{u}}_{\min }\right)\right]^{\mathrm{T}}
$$

Here, $\Delta t$ is the sample interval of the optimizer and $(\boldsymbol{u}, \dot{\boldsymbol{u}})_{\min , \max }$ are present actuator limits and actuator rate limits, respectively.

As seen above, the allocation requires that the Jacobian of $\boldsymbol{f}(\boldsymbol{u})$ and $\boldsymbol{\eta}(\boldsymbol{u})$ can be calculated. To assure this, a rather simple tyre model based on Magic Formula characteristics, non-linear load dependency and coupling between lateral and longitudinal force is used. Tyre and wheel dynamics are excluded and the contact forces are given as analytic functions of $\tau_{i}, \delta_{i}$ and $f_{z i}$, which makes it possible to automatically generate the required partial derivatives.

\section{Evaluation}

The suggested GCC structure is implemented in Modelica [11] as accausual differential-algebraic equations using Dymola [12]. This makes it possible to do automatic inversion, which will be of even greater use in view of later incorporation of more sophisticated inverse models.

As an illustration, a mid-sized four-wheeled passenger car is set up with different actuator combinations to investigate their effect on the performance. As shown in Table 1, actuators are sorted after type, in this case $\delta, \tau$ and $f_{z}$. A pair is chosen from each group for the evaluation, $(A, B),(1,3)$ and $(0, I)$, giving eight combinations from front axle steering and velocity control $(\mathrm{A}, 1,0)$ to front and rear axle steering, individual drive and brake and variable tyre load distribution (B,3,I).

Note that for all configurations, the very same GCC structure and tuning parameters are used, except for the necessary straight forward adaption of the matrix $\mathbf{C}$ representing the configuration-immanent constraints. Note also that a precise reference motion is given such that all configurations may directly 
be compared without considering any driver (human) related property.

For the given trajectory, the reference side slip angle $\beta_{r e f}$ is calculated by a perfectly inverted single track model [7] whose parameters match both the GCC controlled vehicle and the inverse model. To not give the rear axle steered configurations extra advantage, the inverse single track model has only front axle steering. All reference values in the simulation plots are taken from this reference model.

The selected maneouvre is cornering and braking on a $\mu$-split surface. After straight driving at $22 \mathrm{~m} / \mathrm{s}$ for $0.5 \mathrm{~s}$, the vehicle enters a curve to the left with radius $R=80 \mathrm{~m}$, giving $a_{\text {lat }} \approx 6 \mathrm{~m} / \mathrm{s}^{2}$, and at $t=5 \mathrm{~s}$, braking with $a_{\text {long }}=-$ $5 \mathrm{~m} / \mathrm{s}^{2}$ is applied while continuing the cornering. While the single track model uses $\mu=1$, the $\mu$-values of the vehicle and the inverse model used are set $\mu_{1,3}=1.3$ for the inner wheels and $\mu_{2,4}=0.8$ for the outer wheels.

The performance of the different configurations are shown in Figure 2. Vehicle motion and the utilisation $\boldsymbol{\eta}$ of tyre grip potential are displayed where $\boldsymbol{\eta}$ is represented by $\eta_{\text {mean }}=|\boldsymbol{\eta}|_{2}$ and $\eta_{\text {diff }}=\left|\boldsymbol{\eta}-\eta_{\text {mean }} \mathbf{1}\right|_{2}$.

As expected, the biggest difference on the lateral velocity, $v_{y}$, occurs between vehicle configurations with and without rear axle steering, B and A configurations respectively. The A configurations do not manage to keep the preferred $v_{y}$ even during pure cornering (no braking) due to the split $\mu$ conditions, but it can be seen that especially the variable tyre load distribution can improve performance at this stage.

Although a small difference can be spotted for $v_{y}$ between the different $\mathrm{B}$ configurations, the greatest difference is here instead tyre potential usage, $\eta_{\text {mean }}$. While all A configurations reach a peak value of $0.7-0.73,(\mathrm{~B}, 1,0)$ and $(\mathrm{B}, 1, \mathrm{I})$ peaks at about 0.82 . Adding additional torque distribution reduces $\eta_{\text {mean }}$ to about 0.74 which is in parity with the A configurations.

Notable is also that all A configurations have a dip in $a_{y}$ and $v_{y}$ at $t=$ $5 \mathrm{~s}$ because of the additional yaw moment when the braking starts. The $\mathrm{B}$ configurations avoid this by adding an extra steering angle at the rear wheels while the A configurations need to have a higher slip angle than $\beta_{\text {ref }}$ to be able to generate enough side force at the rear axle.

Configuration (A,1,I) shows also a seemingly odd behaviour at $t=7 \mathrm{~s}$ where $\eta_{\text {mean }}$ and $\eta_{\text {diff }}$ suddenly increases. This is in fact due to the circumstance that the rear inner wheel just is about to lose contact with the ground which limits the load distribution.

\section{Concluding discussion}

This work proposes a unique combination of powerful components from control theory for a modular and transparent synthesis of GCC which is directly 

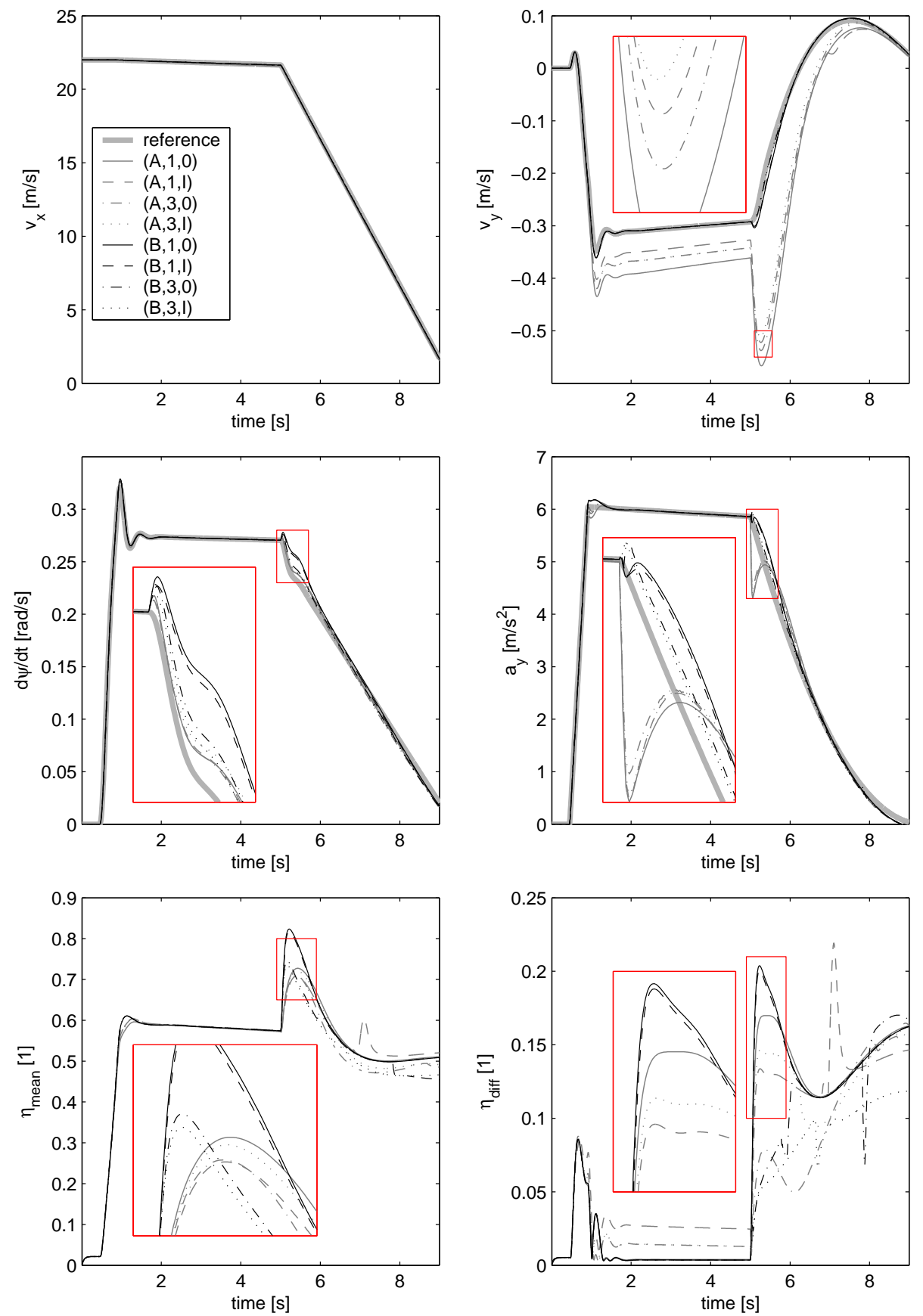

Figure 2. Comparison of performance of some configurations specified in Table 1. Plotted variables are from top left towards the right and bottom; longitudinal speed, lateral speed, yaw rate, lateral acceleration, mean value of grip usage and mean value of grip usage variation. The windows show magnifications of interesting regions. 
applicable to a great variety of vehicle actuation configurations. It gives both a base for GCC design and a structured way to compare different configurations. In practice, the transparency can also be exploited for automatic on-board reconfiguration in case of actuator hardware failures.

To handle the additional degrees of freedom for an inverted over-actuated vehicle, linearization of the allocation problem is suggested. This is reasonable in view of real world actuator position and rate limitations. Moreover, it provides a feasible solution within a limited number of iterations and is thus applicative for real time implementation. At each time step, a least squares optimal solution in the reachable vicinity of the present actuator states is found. This results in smooth actuator commands and avoids jumps between local optima which otherwise may occur for non-convex nonlinear optimization problems.

However, it is important to recall that extending controllability makes it possible to redesign components such as tyres which opens up for other benefits. This method is thus suitable when choosing between existing concepts that fit in the model description. For innovation, human imagination is still needed.

\section{Future work}

The presented work serves as an illustration of a generic global chassis control structure. Some examples of possible extentions from the results presented in this work are presented below.

- This presented evaluation is based on control of the vehicle's horizontal motion. To improve comfort and performance on non-smooth roads, roll, pitch and bounce motion could be included, improving also the dynamic load transfer.

- Additionally, the number of possible actuators could be extended to include camber control and aerodynamic features such as wings to improve down force.

- In this work, $\boldsymbol{f}(\boldsymbol{u})$ and $\boldsymbol{\eta}(\boldsymbol{u})$ are derived by a simple model. This gives fairly compact expressions of the partial derivatives which is believed to be suitable for on-board control. By using tools with powerful symbolic manipulations, Jacobians of more advanced tyre models could be directly formed which would be suitable for concept comparison and validation of the on-board models.

- For on-board use, the inversion is in need of parameters that might be difficult to estimate, such as road adhesion. These will in practice also be given with a certain delay and uncertainty, and thus the effect of this on the performance must be investigated further.

- Limit handling needs to be further investigated. In the case of tyre force sat- 
uration anti-windup measures need to be taken, since IDOB control exhibits integral action.

\section{Acknowledgements}

The authors would like to thank Martin Otter for providing the constrained least squares optimization routine from [10] including his kind support. The first author would also like to thank the Swedish Research Programme "Gröna Bilen/FCHEV" for financial support.

\section{REFERENCES}

[1] Beiker S.: Verbesserungsmöglichkeiten des Fahrverhalten von Pkw durch zusammenwirkende Regelsysteme. Fortschritt-Berichte VDI, Reihe 12, Nr. 418, (2000).

[2] Smakman, H.: Functional integration of slip control with active suspension for improved lateral vehicle dynamics. Herbert Utz Verlag, ISBN 3-89675-704-0, (2000).

[3] Trächtler A.: Integrierte Fahrdynamikregelung mit ESP, aktiver Lenkung und aktivem Fahrwerk. Automatisierungstechnik 53:11-19, (2005)

[4] Orend R.: Modelling and control of a vehicle with single-wheel chassis actuators. Proc. of 16th IFAC World Congress, Prague, (2005).

[5] Andreasson J., Fredriksson, J., Laine L.: Evaluation of a generic vehicle motion control architecture. Proc. of 30th Congress of FISITA, (2004).

[6] Fredriksson, J., Andreasson J., Laine L.: Wheel force distribution for improved handling in a hybrid electric vehicle using nonlinear control, Proc. of 43rd IEEE Conference on Decision and Control, Bahamas, (2004).

[7] Bünte T, Sahin A, Bajcinca N.: Inversion of vehicle steering dynamics with Modelica/Dymola. Proc. of 4th International Modelica Conference, Hamburg, (2005).

[8] Bajcinca N., Bünte T.: A novel control structure for dynamic inversion and tracking tasks. Proc. of 16th IFAC World Congress, Prague, (2005).

[9] Adams R., Banda S.: Robust flight control design using dynamic inversion and structured singular value synthesis. IEEE Transactions on Control Systems Technology, 1(2):80-92, (1993).

[10] Schreiber G., Otter M., Hirzinger G.: Solving the singularity problem of non-redundant manipulator by constraint optimization. In IROS 1999, Kyongju, Korea (pp. 1482-1488), (1999).

[11] Modelica, URL: http://www.modelica.org/

[12] Dymola, URL: http://www.dynasim.se/ 